\title{
INTEROPERABILITY TESTS BETWEEN IFC CERTIFIED SOFTWARE FOR OPEN BIM BASED QUALITY ASSURANCE
}

\author{
$\underline{\text { Jung Sik Choi }}{ }^{1}$ and In Han $\mathrm{Kim}^{2} *$ \\ ${ }^{1}$ Senior Researcher, buildingSMART Korea, Seoul, Korea \\ ${ }^{2}$ Professor, Department of Architecture, Kyung Hee University, Yongin, Korea \\ *Corresponding author (ihkim@khu.ac.kr)
}

\begin{abstract}
The construction industry consists of various and massive architectural information as an architectural process includes a variety of design stages with cooperation of many disciplines. A Building Information Modeling (BIM) serves as a shared knowledge resource for information about a facility forming a reliable basis for decisions during its lifecycle from inception onward. buildingSMART has developed Industry Foundation Classes(IFC) standard for interoperability of BIM based information. Several software tools are already IFC-compliant. However, there are a lot of problems related to efficient interoperability of software owing to the lack of guide regarding the software usage and insufficiency IFC interfaces of software. The purpose of this study is to suggest efficient IFC support interface development methodologies of existing IFC certified software. The scope of this study is interoperability test for open BIM based quality assurance. To achieve this purpose, the authors have classified subject of open BIM based quality assurance and investigated IFC certification process and certified software. In addition, the authors have exported and imported via IFC files using different IFC interfaces in the software for interoperability of architectural objects and their properties. Finally, the authors have suggested IFC support interface development methodologies and hereafter direction for efficient interoperability of IFC certified software based on open BIM
\end{abstract}

Keywords: Building Information Modeling (BIM), Industry Foundation Classes (IFC), Interoperability, Quality Assurance, IFC Certified Software

\section{BIM}

A Building Information Modeling (BIM) is a digital representation of physical and functional characteristics of a facility. The construction industry consists of various and massive information as a construction process includes a variety of stages with cooperation of many disciplines. BIM applies parametric technology and intelligent building objects (wall, slab, window, door, etc.) can represent property (function, structure, usage, regulation information). Therefore, BIM is possible application in various disciplines.

\section{QUALITY ASSURANCE BASED ON OPEN BIM}

Quality Assurance (QA) can be divided according to target and subject of works as following (Table 1).
Table. 1 Classification of Quality Assurance based on Open BIM

\begin{tabular}{|c|c|c|}
\hline $\begin{array}{l}\text { QA for Physical } \\
\text { Information }\end{array}$ & $\begin{array}{l}\text { QA for Logical } \\
\text { Information }\end{array}$ & Data Quality \\
\hline $\begin{array}{l}\text { - Required shape } \\
\text { expression for } \\
\text { individual } \\
\text { components } \\
\text { - Suitable location } \\
\text { for individual } \\
\text { field's models } \\
\text { - Clash between } \\
\text { spaces for } \\
\text { individual field's }\end{array}$ & $\begin{array}{l}\text { - Suitability of } \\
\text { required } \\
\text { information for } \\
\text { individual } \\
\text { components (based } \\
\text { current } \\
\text { law/regular) } \\
\text { - Circulation system } \\
\text { for fire projection } \\
\text { and egress }\end{array}$ & $\begin{array}{l}\text { - Suitability of } \\
\text { shape expression } \\
\text { method } \\
\text { - Validation of } \\
\text { inputting data } \\
\text { properties }\end{array}$ \\
\hline & हैan & 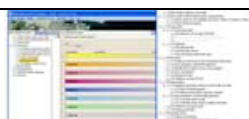 \\
\hline 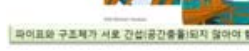 & 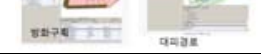 & \\
\hline
\end{tabular}


3. INTEROPERABILITY TESTS BETWEEN IFC CERTIFIED SOFTWARE

In this study, the authors have progressed interoperability tests as following.
- Architectural object: wall, window, door, slab, space(included geometry and property)

- Extension of property information that apply Property set(PSET): Pset_DoorFireResistance

Table. 2 Results and Problems of interoperability tests (Architectural object: Wall, Generated in Revit Architecture)

\begin{tabular}{|c|c|c|c|c|c|c|}
\hline \multicolumn{2}{|r|}{ Wall } & Orign(*.rvt) & ArchiCAD & Revit Architecture & Digital Project & SMC \\
\hline Geometry & $\begin{array}{l}\text { 1.Center Line } \\
\text { 2 Left Line } \\
\text { 3. Right Line } \\
\text { 4. Curve } \\
\text { 5. L-connections } \\
\end{array}$ & & & & & \\
\hline Property & $\begin{array}{l}\text { A. Type Name } \\
\text { B. Material }\end{array}$ & $\begin{array}{l}\text { A-1. Family: } \\
\text { System Family: } \\
\text { Basic Wall } \\
\text { A-2. Type : } \\
\text { 1. Center_Line_Wall } \\
\text { 2. Left_Line_Wall } \\
\text { 3. Right_Line_Wall } \\
\text { 4. Curve_Wall } \\
\text { 5. L-connection_Wall } \\
\\
\text { B. Structure : Concrete }\end{array}$ & $\begin{array}{l}\text { A. ID : } \\
\text { 1. Basic Wall:Cent } \\
\text { 2. Basic Wall:Left } \\
\text { 3. Basic Wall:Righ } \\
\text { 4. Basic Wall:Curv } \\
\text { 5. Basic Wall:L-co } \\
\\
\text { B. Cut Fill : Concrete }\end{array}$ & $\begin{array}{l}\text { A-1. Family: } \\
\text { System Family : Basic Wall } \\
\text { A-2. Type : } \\
\text { 1. Basic Wall: } \\
\text { Center_Line_Wall:135892 } \\
\text { 2. Basic Wall: } \\
\text { Left_Line_Wall:135721 } \\
\text { 3. Basic Wall: } \\
\text { Right_Line_Wall:135760 } \\
\text { 4. Basic Wall: } \\
\text { Curve_Wall:135797 } \\
\text { 5. Basic Wall: } \\
\text { L-connections_Wall:135824 } \\
\text { B. Structure : Concrete }\end{array}$ & $\begin{array}{l}\text { A. Type: } \\
\text { 1. Basic Wall: } \\
\text { Center_Line_Wall:130035 } \\
\text { 2. Basic Wall: } \\
\text { Left_Line_Wall:130232 } \\
\text { 3. Basic Wall: } \\
\text { Right_Line_Wall:130361 } \\
\text { 4. Basic Wall: } \\
\text { Curve_Wall:131423 } \\
\text { 5-1. Basic Wall: } \\
\text { L-connectionsWall:131423 } \\
\text { 5.2. Basic Wall: } \\
\text { L-connections-Wall:131487 } \\
\\
\text { B. Material: Concrete }\end{array}$ & $\begin{array}{l}\text { A. Name : } \\
\text { 1. Basic Wall: } \\
\text { Center_Line_Wall:135892 } \\
\text { 2. Basic Wall: } \\
\text { Left_Line_Wall:135721 } \\
\text { 3. Basic Wall: } \\
\text { Right_Line_Wall:135760 } \\
\text { 4. Basic Wall: } \\
\text { Curve_Wall:135797 } \\
\text { 5. Basic Wall: } \\
\text { L-connections_Wall:135824 } \\
\\
\text { B. Material : Concrete }\end{array}$ \\
\hline & Problems & & $\begin{array}{l}\text { ID have limitation } \\
\text { (15 characters) }\end{array}$ & $\begin{array}{l}\text { Output form of Type value is } \\
\text { different }\end{array}$ & $\begin{array}{l}\text { Name value is indicate in Part } \\
\text { Number }\end{array}$ & $\begin{array}{l}\text { Output form of Name value } \\
\text { is different }\end{array}$ \\
\hline
\end{tabular}

Table. 3 Results and Problems of interoperability tests (PSET, Generated in Revit Architecture)

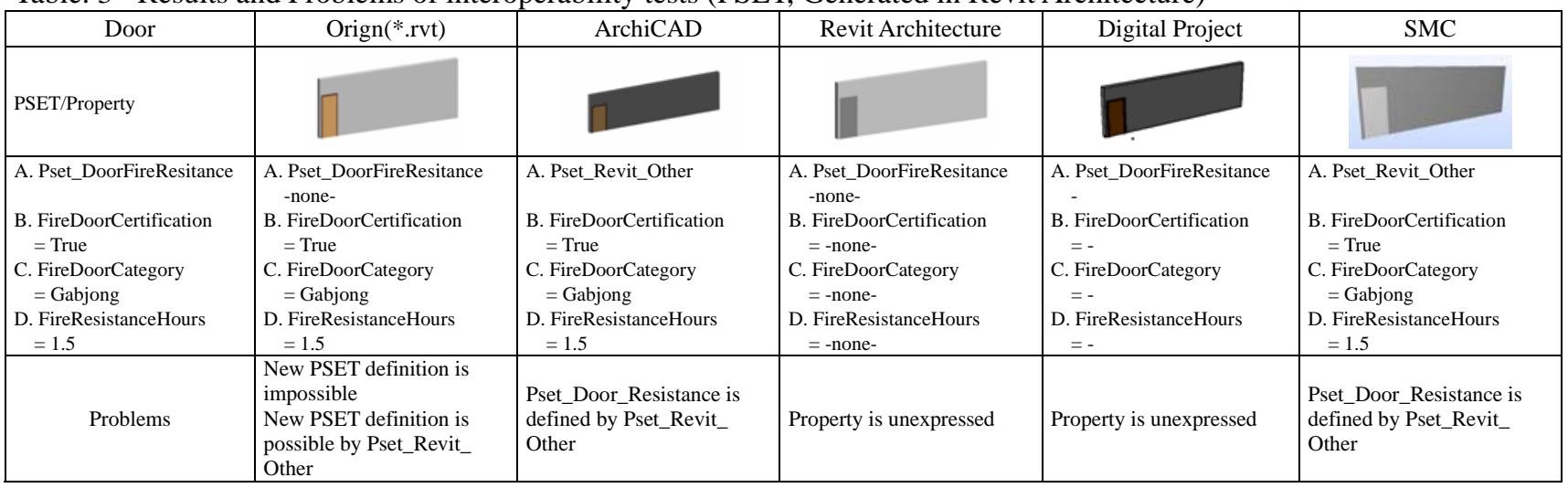

\section{ACKNOWLEDGEMENT}

This research was supported by a grant(code\#09 R\&D A01) from Cutting-edge Urban Development Program funded by the Ministry of Land, Transport and Maritime Affairs of Korean government.

\section{REFERENCES}

[1] I. Kim, “The History and Concept of BIM”, Review of Architecture and Building Science, Vol. 54(1), pp. 16-21, 2010.
[2] A. Kiviniemi, V. Tarandi, J. Karlshoj, H. Bell, O. J. Karud, Review of the Development and Implementation of IFC compatible BIM, Erabuild Report, 2008.

[3] J. Choi, I. Kim, “Application Status of Domestic Architectural Industry of Open BIM and Development Direction”, Transactions of the Society of CAD/CAM Engineers, Vol. 14(6), pp. 355-362, 2009.

[4] Ministry of Land Transport and Maritime Affairs, National Architectural BIM Guide, 2010.

[5] Super-Tall Buildings R\&BD Center, Land-Transport and Maritime R\&D Report, 2010. 